\title{
COMMENT
}

\section{Unified understanding of MS disease is required for drug development}

Timothy Coetzee ${ }^{1}$ and Alan J Thompson ${ }^{2}$

${ }^{1}$ National Multiple Sclerosis Society, 733 Third Avenue, New York, NY 10017 USA

${ }^{2}$ Faculty of Brain Sciences, University College London, 149 Tottenham Court Road London W1T 7NF , UK

Correspondence:

Alan J Thompson

Email: alan.thompson@ucl.ac.uk

Title character count: 90

Standfirst word count: 45

Word count: 1286

References: 5 


\section{Standfirst}

The treatment of multiple sclerosis (MS) has evolved remarkably over the last 25 years. This

progress has been enabled by advances in research, drug development, and active engagement of the scientific community with regulatory authorities. However, an inconsistent approach to MS disease courses could negatively impact the drug development process. 
The treatment of multiple sclerosis (MS) has undergone a remarkable transformation over the last 25 years. Beginning with the approval of IFN $\beta 1 b$ for relapsing remitting MS (RRMS) in 1993, the armamentarium of treatments for MS has grown; now fifteen disease-modifying therapies are available and several others are approved for the treatment of specific symptoms. No other neurological disease has witnessed such remarkable progress, which is an outcome due in large part to a focus on understanding disease mechanisms and on the development of treatments that impact two clinical manifestations of the disease: relapses and disability accumulation. Although none of the available treatments can completely halt relapses and have small effects on the accumulation of disability, recent advances point to a potential future where clinicians can apply a precision medicine approach and achieve considerable outcomes for people with relapsing forms of MS.

The approval of the first treatment for primary progressive MS (PPMS), ocrelizumab in the United States, Europe and Australia, is a much needed development for individuals with this form of the disease $^{1,2}$. Ocrelizumab and IFN $\beta 1 \mathrm{~b}$ offer hope for additional therapeutic advances for progressive MS. There are good grounds for optimism. Patient organizations, the scientific community and the pharmaceutical industry are increasingly focused on the challenges of progressive MS. Although critical barriers exist, such as gaps in scientific knowledge, financial constraints, and regulatory concerns, there is momentum and a collective will to overcome these challenges. Despite this optimism, an issue associated with the approval of ocrelizumab warrants careful consideration; a potential misalignment of stakeholder perspectives regarding progressive MS disease course descriptors, and their application in clinical trials and drug development. 
Prior to 1996, a variety of clinical descriptions were used to characterize patient populations. This changed with the introduction of standardized definitions for the clinical course of MS: RRMS, PPMS, Secondary Progressive MS (SPMS), and Progressive-Relapsing MS (PRMS).

Although the 1996 standardized clinical course descriptors were useful as they enabled more effective communication between patients and clinicians and brought consistency in describing patient populations for clinical research studies, these descriptors were not specifically tied to the pathophysiological mechanisms of disease. A revision of MS descriptors in 2013 took the step of linking phenotypes to disease mechanisms by taking into account the presence of disease activity (relapse occurrence or lesion activity detected by CNS imaging) and/or progression of disability over a given time period. In addition, the 2013 descriptors distinguished RRMS from the progressive forms of the condition and, simultaneously emphasized the many similarities between PPMS and SPMS $^{3}$. However, the authors of these revisions did not advocate combining PPMS and SPMS into one definition, given that there remain some important distinctions between the two forms of progressive MS.

Although the 2013 revision of MS disease course descriptors brought clarity to important gaps in communication and research, this clarity has not extended to the regulatory authorities. Instead, there appears to be a growing divergence in perspectives between the Food and Drug Administration (FDA) and the European Medicines Agency (EMA) on this topic.

At present the European Medicines Agency (EMA) considers the subtypes of MS disease as distinct entities, whereas the FDA appears to view the disease subtypes as part of a continuum of one disease $^{4}$. Although the FDA has not issued specific guidance on this topic, the agency's current 
perspectives are aptly illustrated in the publicly available summary of the ocrelizumab new drug application. In discussing the relapsing and primary progressive forms of MS, the agency wrote: “... It is true that our thinking in the Division regarding the degree of relatedness has evolved ... Previous advice was based on our perception of the understanding of the scientific community and our own understanding concerning the independence of the pathophysiological mechanisms underlying the forms of MS. We have evolved our thinking from our previous position, consistent with a contemporary understanding of MS. Our judgment is that these forms of MS are related, and the Division is prepared to accept PPMS and RRMS populations as mutually supportive." 5

The apparent differences between the two agencies are reflected in their approval statements - with the FDA approving ocrelizumab for PPMS, whereas the EMA approval is for 'early' PPMS - a descriptor not previously used by the community ${ }^{2}$.

The diverging viewpoints of the FDA and EMA could be perceived as a reflection of reasonable debate in the scientific community. However, given the global impact of these two regulatory authorities, a misalignment of their perspectives on a central issue such as MS disease courses could have substantial consequences on clinical development programs in both the academic and industry sector. Researchers and decision-makers could potentially experience uncertainty when deciding which regulatory view to follow in developing their programs. Drug discovery is a challenging effort that requires considerable intellectual and financial commitment by stakeholders. Uncertainty, especially in regulatory matters, adds to this challenge and could introduce an additional disincentive for stakeholders to focus effort and investments in progressive MS. 
A misalignment in perspectives between the regulators on the MS disease courses could also drive the academic and pharmaceutical community towards a focus on treating a disease label - RRMS, PPMS, early PPMS and SPMS - rather than targeting the underlying disease mechanisms.

Considerable progress has been made in distinguishing the inflammatory and neurodegenerative components of MS, although their relationship is still not well understood, and inflammatory and neurodegenerative processes may exist in parallel. Despite this, this knowledge is critically important as we consider underlying mechanisms, with the aim of providing targets for effective treatment. Any move away from this position has the potential to create confusion and thereby delay the development of new treatments.

It is imperative that all stakeholders remain mindful of the fact that MS disease progression is the major determinant of disability, costs and quality of life and that understanding the mechanisms that underlie progression is the major obstacle to the development of effective treatments. Moreover, we cannot forget that these disease descriptors are affixed to individuals, thus, we should consider the implications of this debate on both the patient's treatment and their individual wellbeing.

Although the points discussed here have focused on the FDA and EMA, they are also of relevance to the MS patient, researcher, clinical, and pharmaceutical community. The key question is how will we, as a community, respond? It would be easy to criticize the regulators and demand solutions from them. The reality is that the views of the regulatory authorities are influenced by ongoing debate within the academic community, as well as their interactions with the pharmaceutical sector.

The onus falls on the MS scientific and patient community to lead a global discussion on disease mechanisms that underpin the course of disease, to inform clinical research strategies, and regulatory 
policies and perspectives. Such an effort must provide a clear consensus, based on what is already

known and determine the fundamental questions that need to be answered to provide the necessary clarity going forward. In addition, regulatory authorities should engage the scientific and patient communities in discussions to gauge opinions on disease mechanisms. Such discussions may be challenging to create, but they are the only way to bring a greater transparency to the processes by which the regulatory authorities develop their thinking and policy on such essential matters.

A failure to engage in these collective efforts will lead to a continuation of parallel perspectives in the academic, industry, and regulatory sectors, which could create confusion and the duplication of effort. Without focus and action we risk illogical situations, such as individuals with RRMS and PPMS having access to treatments, but people with SPMS being relegated to a therapeutic desert. Such a circumstance is unacceptable and requires concerted action by the global patient and research communities.

AJT acknowledges support from the UCL/UCLH National Institute of Health (NIHR) Biomedical Research Centre (BRC).

Bibliography

1 Food and Drug Administration. FDA approves new drug to treat multiple sclerosis, https://www.fda.gov/newsevents/newsroom/pressannouncements/ucm549325.htm (2017).

2 European Medicines Agency. New medicine for multiple sclerosis, http://www.ema.europa.eu/ema/index.jsp?curl=pages/news and events/news/2017/11/n ews detail 002847.jsp\&mid=WC0b01ac058004d5c1 (2017).

3 Lublin, F. D. et al. Defining the clinical course of multiple sclerosis: the 2013 revisions. Neurology 83, 278-286, doi:10.1212/wnl.0000000000000560 (2014).

4 European Medicines Agency. Guideline on clinical investigation of medicinal products for the treatment of Multiple Sclerosis, (2015).

$5 \quad$ Food and Drug Administration. Ocrelizumab Summary Review, https://www.accessdata.fda.gov/drugsatfda docs/nda/2017/761053Orig1s000SumR.pdf (2017). 


\section{Competing interests statement}

AJT is chair and TC a member of the International Progressive MS Alliance Scientific Steering Committee

\section{Author contributions}

AJT and TC wrote the article. Both authors contributed to the literature review, the discussion of content, and review and editing of the manuscript.

\section{ONLINE ONLY}

\section{Subject tags}

Health sciences / Diseases / Neurological disorders / Multiple sclerosis

[URI /692/699/375/1666]

Health sciences / Health care / Drug regulation

[URI /692/700/155] 